\title{
A novel medical protocol to treat uterotubal spasm during Essure hysteroscopic sterilization: a pilot study
}

This article was published in the following Dove Press journal:

Open Access Journal of Contraception

19 June 2012

Number of times this article has been viewed

\author{
Hector O Chapa' \\ Gonzalo Venegas ${ }^{2}$ \\ 'Women's Specialty Center, \\ Dallas, TX, USA; ${ }^{2}$ Clinical Faculty, \\ Department ObGyn Methodist \\ Medical Center, Dallas, TX, USA
}

Correspondence: Hector O Chapa 2042 Marydale Road, Dallas,

TX, 2042, USA

Tel + I 2143348I I6

Email chapamd@gmail.com
Objective: To evaluate sublingual hyoscyamine $(0.125 \mathrm{mg})$ as a uterotubal spasm reliever to increase successful bilateral, first-attempt Essure microinsert placement.

Study design: Prospective cohort study as pilot clinical investigation of study medicine. Setting: An inner-city Dallas, TX obstetrics and gynecology office practice.

Materials and methods: The study period was January 1, 2008 to July 1, 2010. Patients requesting sterilization were offered Essure under local anesthesia (office setting). Those declining were referred for operative laparoscopy. Patients accepting office sterilization were offered study participation. Study patients noted to have intraoperative uni/bilateral uterotubal spasms preventing cannulation were given one sublingual hyoscyamine $(0.125 \mathrm{mg})$ tablet. Primary endpoint: spasm alleviation and successful tubal cannulation (bilaterally). Secondary endpoint: percentage of bilateral tubal occlusions documented by follow up hysterosalpingogram (12 weeks), and adverse events possibly related to medication.

Results: Within the study period, 316 patients underwent office sterilization (local anesthesia); 21 had unilateral tubal spasm preventing cannulation (6\%). Spasm was relieved in 17/21 (80\%) after hyoscyamine, allowing for microinsert placement. The mean time from attempted cannulation to medication was 9 minutes (8.3-10.5 minutes), and the mean time for spasm resolution was 53 seconds (49-72 seconds). At follow-up confirmation testing, all 17 who were hyoscynamine responders were found to have bilateral tubal occlusion as well as proper microinsert location. The 4/21 hyoscyamine nonresponders underwent a second unilateral attempt 48-72 hours later. Fifty percent $(n=2)$ had successful placement, leaving two for alternative care. Rapid pulse was reported by $1 / 21(4.7 \%) 5$ minutes postmedication.

Conclusion: Essure sterilization has a high bilateral, first-attempt success rate. Hyoscyamine may aid in spasm relief and increase single procedure, bilateral placement success rate further. Keywords: hysteroscopic sterilization, Essure, tubal spasm, hyoscyamine

\section{Background}

According to the Food and Drug Administration (FDA) postapproval study for the Essure $^{\circledR} 305$ device (Conceptus Incorporated, Mountain View, CA), the first-attempt bilateral placement rate for the current Essure system increased from $94.6 \%$ to $96.9 \%$, and the hysteroscopic time decreased from 13 to 9 minutes ${ }^{1,2}$ among the 94 participating physicians treating 612 patients. Panel and Grosdemouge reported success with Essure among $96.7 \%$ of the 492 patients included in their prospective multicenter study in 2006. ${ }^{3}$ Success was defined as bilateral placement of the Essure (on the first or second attempt), or unilateral placement with a history of a unilateral salpingectomy, or confirmation of occlusion of the second fallopian tube via hysterosalpingogram. 
According to published data, factors impeding firstattempt, bilateral placement from being possible include uterine anomalies and proximal tubal occlusion, tortuosity, spasm, or stenosis. ${ }^{4,5}$ In an effort to reduce second attempts, we sought to investigate the off-label use of hyoscyamine to treat procedural uterotubal spasms. Our study hypothesis was that the antispasmatic effect of the medication would increase first-attempt, bilateral placement rates.

\section{Materials and methods}

All patients accepting office hysteroscopic sterilization were offered study participation. The study protocol was reviewed and approved by the Ethical Review Board at Women's Specialty Center Dallas (WSC) (Dallas, TX). Patients were informed of the off-label use of the study medication, and they were also informed of its FDA-approved indication. Per standard protocol at WSC, and in accordance with the manufacturer's instructions for use, ${ }^{7}$ slow forward pressure was exerted at cannulation during a suspected tubal spasm preventing microinsert placement. For participants who agreed to participate in the study, a nurse administered sublingual hyoscyamine (one tablet, $0.125 \mathrm{mg}$ ) after a maximum of 10 minutes of attempted cannulation. This occurred while the operator continued to keep proper pressure on the attempted tubal cannulation. If participants did not wish to take part in the study, or if no response was noted to the study drug after 5 minutes, the patients had the procedure terminated and were offered either laparoscopic sterilization or a reattempt at the hysteroscopic approach within 48 to 72 hours later.

Patients were not permitted to participate in the medication trial if they had a current or past history of glaucoma, obstructive uropathy, or uncontrolled hypertension.

All procedures were performed by the same Essurecertified attending physician under local paracervical block (diluted 1\% mepivacaine). All patients were sublingually administered $30 \mathrm{mg}$ of ketorolac for 25-30 minutes prior to the procedure. In accordance with the WSC protocol, all patients received oral medroxyprogesterone acetate $(10 \mathrm{mg})$ twice a day for 10 days preprocedure (endometrial pretreatment) with sterilization attempted 1-2 days after endometrial preparation. This occurred regardless of menstrual cycle timing.

Although not a randomized trial, we hypothesized that the sample size for recruitment would be approximately 18, and that the results would show a $50 \%$ reduction/resolution. This was based on the results of our internal 2007 experience of tubal spasm during office-based Essure placement, assuming 80\% power and utilizing a significance level of 0.05 . To calculate sample size, we used data collected from 50 patients who had previ- ously undergone Essure placement (office-based). We noted an average time of 5.2 minutes for spontaneous uterotubal spasm resolution (either by constant Essure cannula pressure placement or after hysteroscope removal with wait time to reinsert/ reattempt). We therefore set the maximum anticipated time for spasm resolution as 4 minutes (assuming the time for the drug effect to be 1 minute, with a reduction in spasm time averaging approximately 5 minutes; ie, a maximum of 5 minutes for spontaneous spasm resolution, with medication effect onset occurring at 1 minute, and a 4-minute time difference as delta). For these patients, the minimum time for bilateral placement was 4.3 minutes; maximum time for bilateral placement was 11 minutes, with a mean time of 8.4 minutes. Procedure time was defined as time from hysteroscope insertion into the uterine cavity until final hysteroscope removal for procedure termination. Using the calculated standard deviation (SD) from these 50 patients' mean procedure time ( 4.2 minutes), and a $\mathrm{K}$ value of 8 for $80 \%$ power, we arrived at the decision that a total 20 patients were needed in each arm of the study.

$$
\mathrm{N}=2(\mathrm{k}) /(\Delta / \mathrm{SD})^{2}+1
$$

Statistical analysis was performed with Minitab statistical software (v 1; Minitab Inc, State College, PA). Demographic differences between groups were calculated using rank-sum test for continuous variables and Chi-square test for binary data.

\section{Results}

Beginning on January 1, 2008, we began our pilot study using sublingual hyoscyamine tablets when uterotubal spasm was encountered. As a comparison, we analyzed our prior (2007) 6-month uterotubal spasm rate documented intraoperatively by our group. For that year, we noted a bilateral first-attempt placement rate of $95 \%$, with a $5 \%$ uterotubal spasm incidence preventing completion. Within the study period, 316 patients presented for and underwent office sterilization (local anesthesia). No patients declined study participation after informed consent. Patient characteristics are shown in Table 1. When offered either the laparoscopic or hysteroscopic approach, $100 \%$ of participants chose the hysteroscopic trial as their index procedure. Twenty-one of 316 participants had unilateral tubal spasm preventing cannulation (6\%). Spasm was relieved in 17/21 (80\%) patients after hyoscyamine administration, allowing for microinsert placement. Mean time from attempted cannulation to medication was 9 minutes (8.3-10.5 minutes). Mean time for spasm resolution was 53 seconds (49-72 seconds). Those with intraoperative 
Table I Patient sociodemographics $(\mathrm{n}=316$ total)

\begin{tabular}{|c|c|c|c|}
\hline Factors & $\begin{array}{l}\text { No spasm group } \\
(n=295), n(\%)\end{array}$ & $\begin{array}{l}\text { Spasm group } \\
(n=2 I), n(\%)\end{array}$ & $P$-value \\
\hline $\begin{array}{l}\text { Age (median years), } \\
\text { (range) }\end{array}$ & $\begin{array}{l}39 \\
(35-44)\end{array}$ & $\begin{array}{l}37 \\
(34-43)\end{array}$ & 0.81 \\
\hline \multicolumn{4}{|l|}{ Race } \\
\hline Hispanic & $186(63)$ & $16(76)$ & 0.76 \\
\hline Black & $70(24)$ & $3(14)$ & 0.09 \\
\hline Caucasian & $39(13)$ & $2(10)$ & 0.08 \\
\hline $\begin{array}{l}\text { Uterine size }(\mathrm{cm}) \text {, } \\
\text { median (range) }\end{array}$ & $10(8-I I)$ & $9.5(9-11.4)$ & 0.98 \\
\hline BMI median (range) & $28.3(27.1-29.3)$ & $27.9(27.9-28.8)$ & 0.86 \\
\hline \multicolumn{4}{|l|}{ Parity } \\
\hline$\leq 3$ & $242(82)$ & I8 (86) & 0.81 \\
\hline $4-6$ & $53(18)$ & $3(14)$ & 1.23 \\
\hline$\geq 7$ & 0 & 0 & NA \\
\hline $\begin{array}{l}\text { Scope time } \\
\text { (min), median (range) }\end{array}$ & $7.3(6.4-9.3)$ & I2.5 (9.4-|4.5) & 0.04 \\
\hline
\end{tabular}

Notes: Differences between groups were calculated using rank-sum test for continuous variables and Chi-square for binary data. $P<0.05$, statistically significant Abbreviations: BMI, body mass index; $n$, number of patients; $P$, probability.

tubal spasm had longer mean hysteroscopic operative times ("scope time") than those without $(P=0.04)$.

In the medication responders' cohort $(\mathrm{n}=17)$, hysterosalpingogram (HSG) follow-up revealed bilateral occlusion, proper microinsert location in $100 \%$ (17/17). HSGs for this cohort took place from 11-15 weeks post-Essure placement. The $4 / 21$ hyoscyamine nonresponders underwent a second unilateral attempt $48-72$ hours later. Fifty percent $(n=2)$ had successful placement with no spasm noted, leaving two requiring alternative care. Follow-up HSG for the two patients documented satisfactory placement and occlusion at 13 and 14 weeks, respectively. The patients with unilateral placements (failed Essure bilateral insertions) underwent laparoscopic sterilization within the 2 weeks following the failed second attempt.

Of patients given study medication, 1 participant (4.7\%) reported experiencing a possible drug side effect. The symptom reported was "rapid heart rate" at 5 minutes postadministration. The patient's pulse was 98 on pulse oximeter, and symptoms resolved after 3 minutes. No additional care or attention was required.

\section{Discussion}

Since the introduction of the Essure hysteroscopic sterilization system in 2002, a trend towards this method of female sterilization has been well documented. According to one published report, $97.9 \%$ of female sterilizations were performed by laparoscopic approach in 2002 compared to $0 \%$ via the hysteroscopic method. By 2007, 48.5\% were completed laparoscopically versus $51.3 \%$ by the Essure method. ${ }^{5}$
As mentioned previously, the reported rate of bilateral, first-attempt microinsert placement with the Essure system is $96.9 \% .^{2}$ Miño et al have published their experience based upon 857 treated women. ${ }^{7}$ Insertion was achieved in close to $99 \%$ of their cohort. The investigators described the procedure as difficult in $15 \%$ of the cases, mainly due to anatomical tubal anomalies or tubal spasm. Remarkably, the study women "felt highly satisfied in all cases." Tips at improving placement rates have included good visualization of the tubal ostia, placement during the proliferative phase of the menstrual cycle, and non-steroidal anti-inflammatory premedication. ${ }^{8,9}$ When using the keywords "tubal spasm," "hysteroscopy," "sterilization," and "Essure," we were unable to find any peer-reviewed, published data on medical strategies to treat/prevent uterotubal spasm during the hysteroscopic sterilization procedure. We therefore sought to investigate an existing antispasmodic medication as a pilot protocol to prevent failed bilateral, first-attempt microinsert placements.

Hyoscyamine is one of the principal anticholinergic/ antispasmodic components of belladonna alkaloids. ${ }^{10}$ Current indications for use include the treatment of peptic ulcer, the control of gastric secretion, spasm relief for colitis, spastic bladder, cystitis, pylorospasm, and associated abdominal cramps. Additionally, hyoscyamine is indicated as an adjunctive therapy in the treatment of neurogenic bladder and neurogenic bowel disturbances. ${ }^{10}$ Onset of action depends on dose and route of administration, with sublingual routes having a range of 2-20 minutes for onset. ${ }^{10}$ Although offlabel in its study use, the authors screened patients for morbid contraindications before allowing final study participation. Additionally, the dosage chosen, as a single administered dose, would be theoretically unlikely to promote severe adverse events.

We elected to administer the study drug after the intraoperative diagnosis of uterotubal spasm was made by the inability to forward-insert the device catheter. As per the manufacturer's recommendation, 10 minutes per side of attempted cannulation/placement is suggested for a maximum "scope time" of 20 minutes. ${ }^{7}$ Therefore, our protocol ensured that after the inability to cannulate for a minimum of $5 \mathrm{~min}$ utes, physicians (investigators) had the option to order study drug administration. After 10 minutes of failed cannulation, the study drug was to be administered. Failure to alleviate the spasm was determined at 5 minutes after sublingual use. Our findings indicate a potential role for hyoscyamine use as adjuvant therapy in cases where failed bilateral placement may be an issue. In our cohort of 316 total patients treated by 
hysteroscopic sterilization, a 94\% successful bilateral firstattempt rate was increased to $98.7 \%$ by tubal spasm resolution, leaving only 4 patients requiring a second attempt.

Although not a randomized, blinded study, the value of this investigation lies in this being the only studied medication protocol designed to improve Essure placement rates; however, several questions remain unanswered. The optimum dose, timing, and route of administration of this medication were not addressed by our protocol. Additionally, we cannot estimate how many patients would have had spontaneous resolution with longer expectant management of the spasm. Nonetheless, our protocol adds a significant advantage in these cases since prolonging the hysteroscopic procedure may increase fluid intravasation and patient discomfort. This is the main clinical implication and application of our study results.

We chose 5 minutes as the cut-off time for spasm resolution to be considered as successful. This was based on the expected time for onset of action of the medication. Perhaps by providing a longer wait time to determine medication effectiveness we could have increased our overall success rate. This is another recognized limitation of our design. Regardless, given that this study is the first of its kind, we believe this protocol is successful as a pilot investigation. The authors are planning a randomized, double blinded, placebo controlled trial to further delineate and confirm our preliminary results.

Our study period incidence of uterotubal spasm was $6 \%$, which is slightly higher than our rate of $5 \%$ noted historically, giving us a $94 \%$ bilateral placement rate. This is in contrast to the FDA accepted rate of $96.7 \%$. We cannot exclude physician/observer bias in potentially overestimating how many women were experiencing spasms given that the investigators were not blinded to the protocol. We are aware that blinding the investigators may potentially alter the rate of diagnosis of tubal incidence.

As physicians may be reluctant to adopt these minimally invasive technologies for fear of potential negative health events such as tubal spasm, the value of this proposed study is clear. The knowledge presented here may increase the utilization of hysteroscopic sterilization, thereby offering patients more options and minimizing potential patient risk from elective laparoscopic alternatives. ${ }^{11}$

\section{Disclosure}

Hector O Chapa, MD, serves as a medical consultant to Conceptus, Inc, and sits on the Conceptus Physicians' Advisory Panel. No financial or corporate assistance was provided for the organization, production, or submission of this manuscript. Views reflected in this submission are those of the authors and not of any corporate entity.

\section{References}

1. Conceptus(R) Receives FDA Approval On Essure's Bilateral Placement Rate To $96.9 \%$ [press release]. May 19, 2010. [cited December 4, 2011]. Available from: http://www.reuters.com/article/2010/05/18/idUS226590+18May-2010+BW20100518. Accessed December 4, 2011.

2. Conceptus Essure 305 Instructions for Use [homepage on the Internet]. Mountain View: Conceptus Incorporated [updated 2007; cited December 4, 2011]. Available from: http://www.Essure.com. Accessed December 4, 2011.

3. Panel P, Grosdemouge I. Predictive factors of Essure implant placement failure: prospective, multicenter study of 495 patients. Fertil Steril. 2010;93(1):29-34.

4. Cooper JM, Carignan CS, Cher D, Kerin JF. Microinsert nonincisional hysteroscopic sterilization. Obstet Gynecol. 2003;102(1):59-67.

5. Kerin JF, Munday DN, Ritossa MG, Pesce A, Rosen D. Essure hysteroscopic sterilization: results based on utilizing a new coil catheter delivery system. J Am Assoc Gynecol Laparosc. 2004;11(3):388-393.

6. Shavell V, Abdallah ME, Shade GH Jr, Diamomd MP, Berman JM. Trends in sterilization since introduction of Essure hysterocopic sterilization. J Minim Invasive Gynecol. 2009;16(1):22-27.

7. Miño M, Arjona JE, Cordón J, Pelegrin B, Povedano B, Chacon E. Success rate and patient satisfaction with the Essure sterilisation in an outpatient setting: a prospective study of 857 women. BJOG. 2007;114(6):763-766.

8. Rosen DM. Learning curve for hysteroscopic sterilisation: lessons from the first 80 cases. Aust N Z J Obstet Gynaecol. 2004;44(1):62-64.

9. Nichols M, Carter JF, Fylstra DL, Childers M. A comparative study of hysteroscopic sterilization performed in-office versus a hospital operating room. J Minim Invasive Gynecol. 2006;13(5):447-450.

10. RxList the internet drug index [homepage on the Internet]. New York: WebMD [cited December 05, 2011]. Available from: http://www.rxlist. com/levsin-drug.htm. Accessed December 5, 2011.

11. Fuller J, Scott W, Ashar B, Corrado J. Laparoscopic Trocar Injuries: A report from a US Food and Drug Administration (FDA) Center for Devices and Radiological Health (CDRH) Systematic Technology Assessment of Medical Products (STAMP) Committee. Silver Spring, MD: US Food and Drug Administration; 2003. Available from: http:// www.fda.gov/medicaldevices/safety/alertsandnotices/ucm197339.htm. Accessed January 3, 2011.
Open Access Journal of Contraception

\section{Publish your work in this journal}

Open Access Journal of Contraception is an international, peerreviewed, open access, online journal, publishing original research, reports, reviews and commentaries on all areas of contraception. In addition to clinical research, demographics and health-related aspects, the journal welcomes new findings in animal and preclinical studies

\section{Dovepress}

relating to understanding the biological mechanisms and practical development of new contraceptive agents. The manuscript management system is completely online and includes a very quick and fair peer-review system. Visit http://www.dovepress.com/testimonials.php to read real quotes from published authors. 